\title{
Potential Impact of Rapid Blood Culture Testing for Gram-Positive Bacteremia in Japan with the Verigene Gram-Positive Blood Culture Test
}

\author{
Ken Kikuchi, ${ }^{1,2,3}$ Mari Matsuda, ${ }^{3}$ Shigekazu Iguchi, ${ }^{1,3}$ Tomonori Mizutani, ${ }^{3}$ Keiichi \\ Hiramatsu, ${ }^{3}$ Michiru Tega-Ishii, ${ }^{2}$ Kaori Sansaka, ${ }^{2}$ Kenta Negishi, ${ }^{2}$ Kimie Shimada, ${ }^{2}$ Jun \\ Umemura, ${ }^{2}$ Shigeyuki Notake, ${ }^{4}$ Hideji Yanagisawa, ${ }^{4}$ Hiroshi Takahashi, ${ }^{5}$ Reiko Yabusaki, ${ }^{6}$ \\ Hideki Araoka, ${ }^{6}$ and Akiko Yoneyama ${ }^{6}$ \\ ${ }^{1}$ Department of Infectious Diseases, Tokyo Women's Medical University, 8-1 Kawada-cho, Shinjuku-ku, Tokyo 162-8666, Japan \\ ${ }^{2}$ Sakakibara Heart Institute, 1-16-1 Asahimachi, Fuchu, Tokyo 183-0003, Japan \\ ${ }^{3}$ Department of Infection Control Science, Faculty of Medicine, Juntendo University, 2-1-1 Hongo, Bunkyo-ku, Tokyo 113-8421, Japan \\ ${ }^{4}$ Miroku Medical Laboratory Inc., 659-2 Innai, Saku, Nagano 384-2201, Japan \\ ${ }^{5}$ East-West Diagnostics/Theranostics, San Francisco, CA 94105, USA \\ ${ }^{6}$ Department of Infectious Diseases, Toranomon Hospital, 2-2-2 Toranomon, Minato-ku, Tokyo 105-8470, Japan
}

Correspondence should be addressed to Ken Kikuchi; kikuchi.ken@twmu.ac.jp

Received 18 October 2016; Revised 27 November 2016; Accepted 18 December 2016; Published 20 February 2017

Academic Editor: José Ramón Blanco

Copyright (C) 2017 Ken Kikuchi et al. This is an open access article distributed under the Creative Commons Attribution License, which permits unrestricted use, distribution, and reproduction in any medium, provided the original work is properly cited.

Background. Early detection of Gram-positive bacteremia and timely appropriate antimicrobial therapy are required for decreasing patient mortality. The purpose of our study was to evaluate the performance of the Verigene Gram-positive blood culture assay (BC-GP) in two special healthcare settings and determine the potential impact of rapid blood culture testing for Gram-positive bacteremia within the Japanese healthcare delivery system. Furthermore, the study included simulated blood cultures, which included a library of well-characterized methicillin-resistant Staphylococcus aureus (MRSA) and vancomycin-resistant enterococci (VRE) isolates reflecting different geographical regions in Japan. Methods. A total 347 BC-GP assays were performed on clinical and simulated blood cultures. BC-GP results were compared to results obtained by reference methods for genus/species identification and detection of resistance genes using molecular and MALDI-TOF MS methodologies. Results. For identification and detection of resistance genes at two clinical sites and simulated blood cultures, overall concordance of BC-GP with reference methods was 327/347 (94\%). The time for identification and antimicrobial resistance detection by BC-GP was significantly shorter compared to routine testing especially at the cardiology hospital, which does not offer clinical microbiology services on weekends and holidays. Conclusion. BC-GP generated accurate identification and detection of resistance markers compared with routine laboratory methods for Gram-positive organisms in specialized clinical settings providing more rapid results than current routine testing.

\section{Introduction}

Gram-positive bacteria are the most predominant microorganisms associated with sepsis in healthcare settings and the most prevalent cause of bacteremia in patients with hematopoietic stem cell transplantation $[1,2]$. Enterococcal bacteremia is associated with increased risk of mortality in patients with hematopoietic stem cell transplantation, irrespective of susceptibility to vancomycin $[3,4]$. In cardiology units, infective endocarditis, infectious aneurysm, catheter-related bloodstream infections, or surgical site infections after cardiac surgeries are the major infections of which the most common causative microorganisms are Grampositive cocci [5-10]. In both medical units, early detection of 
Gram-positives and resistant markers is very critical in managing patient care, antibiotic stewardship, and preventing spread of resistant microorganisms.

As early intervention with antimicrobial therapy is associated with improved prognosis with each hour of delay associated with increased mortality, rapid diagnosis is critical [11]. The Verigene Gram-positive blood culture assay (BC-GP) (Nanosphere, Inc., Northbrook, IL) is a sample to result microarray system for identification of common Gram-positive bacteria and major resistance markers directly from positive blood culture. Although a number of studies have previously evaluated BC-GP reporting performance ranging from 92 to $99 \%$ agreement with conventional methodology [12-16], one limitation of previous reports has been that many of the studies have been from the United States as well as countries outside of Japan with only one published report of limited scope in Japan [17].

Genetic variation among bacterial lineages circulating in different geographical regions of the world can affect the sensitivity of molecular assays based on oligonucleotide probes to detect organisms or resistance markers. Studies in Hong Kong and Belgium have reported lower BC-GP performance $[18,19]$.

The purpose of this study was to determine the potential impact on patient management and patient outcome of BCGP in specialized hospitalized settings within the Japanese healthcare environment, which faces a number of challenges. An increasingly aging population and the accompanying burden of increasing overall healthcare costs have challenged the healthcare infrastructure. Despite methicillin-resistant Staphylococcus aureus (MRSA) accounting for over $90 \%$ of the hospital-acquired infections caused by resistant bacteria in Japan [20], outsourcing of clinical microbiology testing or no weekend coverage is common in many healthcare facilities as part of cost-containment. This paper represents the first comprehensive evaluation of BC-GP in Japan to validate its clinical performance. The simulated blood culture study includes a library of well-characterized healthcareassociated MRSA (HA-MRSA), community-acquired MRSA (CA-MRSA), and vancomycin-resistant enterococci (VRE) strains circulating in Japan.

\section{Methods}

BC-GP was evaluated at Toranomon Hospital (TH) and Sakakibara Heart Institute (SHI), in accordance with site-specific institutional review board approved study protocols, during June 26, 2012, to March 6, 2013. TH is an 1168-bed general teaching hospital with a 123-bed hematological care unit for hematopoietic stem cell transplantation performing 140 to 160 hematopoietic stem cell transplants per year. The microbiology laboratory at the hospital operates daily during day hours. SHI is a 320-bed teaching hospital specializing in cardiovascular diseases with a caseload of over 1,500 openheart surgeries per year. The microbiology laboratory in the hospital is operated by an outside commercial reference laboratory. The microbiology laboratory operates during the day shift on weekdays and closed on weekends and holidays.
Blood cultures were performed at SHI using BacT/ ALERT FA bottles and monitoring with BacT/ALERT 3D (bioMérieux, Marcy l'Etoile, France). TH used BACTEC Plus bottles and monitoring with BACTEC 9240 and FX (Becton Dickinson, Franklin Lakes, and NJ). Only one positive blood culture bottle containing Gram-positive cocci or bacilli per patient was included in the study. Two $\mathrm{ml}$ of positive blood culture medium was stored at $-85 \mathrm{C}$ for retesting.

Routine microbiological identification and susceptibility testing of isolates were performed using conventional identification tests such as bile solubility, optochin disk susceptibility, and the MicroScan WalkAway system (Beckman Coulter, Pasadena, CA) at TH and the Vitek 2 system (bioMérieux, Marcy l'Etoile, France) at SHI. Cefoxitin screening for methicillin resistance was performed according to CLSI guidelines [21]. A latex agglutination test for detection of penicillinbinding protein PBP2a was also utilized [22].

BC-GP testing was performed on positive blood culture showing Gram-positive organisms according to the manufacturer's instructions. Briefly, a well-mixed $350 \mu \mathrm{l}$ sample of the blood culture media was pipetted into the sample well of the BC-GP nucleic extraction tray, placed onto the Verigene Processor SP for processing and analysis by the Verigene Reader.

An assessment was performed to determine the difference in time between reporting of results using BC-GP and culture-based identification and antimicrobial susceptibility results for 139 positive blood culture broths. For culturebased results, the time required until generation of the final report was the time between the Gram stain reading and entering of final identification and susceptibility results into the laboratory information system. For BC-GP, the time to result was the time between the Gram stain reading and entering of $\mathrm{BC}-\mathrm{GP}$ results into the laboratory information system.

A challenge set of 208 simulated blood cultures was constructed using type, reference strains, and clinical strains from different geographical regions in Japan to evaluate BCGP. The clinical strains included organisms that presented challenges for commercial identification systems in previous clinical studies at $\mathrm{TH}$ and SHI. In addition, a library of well-characterized HA-MRSA, CA-MRSA, and VRE strains from Japan was also tested. Simulated blood culture studies were performed at Miroku Medical Laboratory (Saku City, Nagano Prefecture, Japan). Two hundred and eight strains were adjusted to a turbidity of approximately $100 \mathrm{CFU} / \mathrm{ml}$ in sterile saline. Three hundred $\mu \mathrm{l}$ was inoculated into BACTEC Plus Aerobic/F bottles containing 8 to $10 \mathrm{ml}$ of human whole blood (blood type O, Tennessee Blood Services, Memphis, TN) for a final inoculum of $30 \mathrm{CFU} /$ bottle. A BACTEC Plus Anaerobic/F bottle was also inoculated for S. pneumoniae and the $S$. anginosus group. Each bottle was incubated in the BACTEC system until a positive signal was generated. If BCGP generated negative results, 11-fold dilution of the blood culture medium using sterile distilled water was retested.

Each of the positive blood culture isolates at the two hospital sites was stored in $10 \%$ skim milk (Difco) at $-85 \mathrm{C}$. Species identification was confirmed using matrix-assisted 
laser desorption ionization-time of flight mass spectrometry (MALDI-TOF MS) (Microflex LT with Biotyper ver. 3.0 software; Bruker Daltonik GmbH, Bremen, Germany) for all strains [23]. If the organism was not identified to the species level by MALDI-TOF MS (score value < 2.0) or identified as Micrococcus, Listeria, Staphylococcus other than S. aureus, Streptococcus other than S. pyogenes, and S. agalactiae, confirmatory testing by PCR-direct sequencing of $16 \mathrm{~S}$ rDNA or $\operatorname{sodA}$ was performed at Juntendo University or Tokyo Women's Medical University [24-26]. Specific-PCR for detecting mecA [27] in all staphylococci and vanA, vanB in all enterococci was performed [28]. The methods used for SCCmec typing of community-acquired or healthcareassociated MRSA used to characterized strains have been previously described $[29,30]$.

Concordance was determined in comparison to results of the reference methods. There was agreement if BC-GP target detection agreed with the reference method at either the genus or the species level. The ninety-five percent confidence intervals $(95 \% \mathrm{CI})$ and the paired $t$-test were determined using GraphPad StatMate (GraphPad Software Inc., San Diego, CA).

\section{Results}

In this study, the overall identification agreement between BC-GP and the reference method was 327/347 (94\%) for prospective blood cultures at two clinical sites and simulated blood cultures. The combined agreement between PCR and BC-GP for mecA detection was 71/73 (97\%) for prospective blood cultures and simulated blood cultures.

The combined identification accuracy from both hospital sites was 129/139 (93\%). For monomicrobial cultures, the identification accuracy was 121/124 (98\%). Agreement between PCR and BC-GP for mecA positivity was 51/53 (96\%). Table 1 shows the results from TH. Overall, 96/104 (92\%) organisms were correctly identified by BC-GP to the species or genus level including detection of resistance genes. As shown in Table 2, total agreement of BC-GP with the reference method at SHI was 33/35 (94\%) organisms.

BC-GP reports the presence of mecA for only $S$. aureus and $S$. epidermidis. In this study, of the 102 staphylococcal strains, 72 were either $S$. aureus or $S$. epidermidis. Discordant results were due to undetectable mecA $S$. epidermidis organisms in polymicrobial cultures containing both mecA positive and mecA negative S. epidermidis. Of the 30 Staphylococcus spp., other than S. epidermidis and S. aureus, 21 (70\%) were positive for $m e c A$, including $2 S$. lugdunensis, which could not be reported as mecA positive by BC-GP.

Table 3 shows the difference in time between the generation of BC-GP results and culture-based final identification and antimicrobial susceptibility results at both hospitals. BCGP results were available at a mean of 28.2 to 51.0 hours before culture-based final identification and susceptibility results at TH. At SHI, BC-GP generated results at a mean of 34.5 to 196.6 hours earlier. In comparing the time to final culturebased identification and susceptibility results at TH and SHI, with the exception of $S$. aureus, results for S. epidermidis and coagulase-negative staphylococci other than $S$. epidermidis, enterococci, and streptococci required significantly $(P<$ 0.05) longer time at SHI (83.3, 123.6, 159.1, and 199.1 hours, resp.) compared to $\mathrm{TH}(40.8,53.9,36.1$, and 53.5 hours, resp.).

Using simulated Gram-positive blood cultures, BC-GP correctly identified 198/208 (95\%) of the organisms (Table 4). Six streptococci (3\%) were either incorrectly identified or identified at the genus level only by BC-GP. With respect to the 4 BC-GP false-negative blood culture bottles, $1 \mathrm{~S}$. pyogenes was correctly identified and $1 S$. mitis generated a positive Streptococcus genus/S. pneumoniae signal following 11-fold dilution of the blood culture medium. The mecA gene was detected in 20/20 (100\%) MRSA organisms representing community-acquired (SCCmec type IIa, IV, and V) and healthcare-associated (SCCmec type I, IIb, III, and nontypeable) strains by BC-GP. BC-GP detected 14/14 (100\%) vanA and 20/20 (100\%) vanB genes in well-characterized VRE strains from previous studies in Japan.

\section{Discussion}

The performance of BC-GP observed in our study was similar to previous reports [12-16, 31-33]. As clinical microbiology services are outsourced or not operating during off-shifts during weekdays and closed on weekends and holidays in many hospitals in Japan, rapid diagnostic testing has significant potential to impact patient care by dramatically decreasing the time to organism identification and antimicrobial susceptibility results.

BC-GP detects mecA in all staphylococci based on measurement of signal intensity; however, reporting is restricted to $S$. aureus and $S$. epidermidis based on an algorithm in which mecA is only reported when $S$. aureus or S. epidermidis is detected by BC-GP. Future versions of BC-GP should consider modification of the algorithm to allow reporting of $m e c A$ detection for staphylococci other than $S$. aureus or $S$. epidermidis as $70 \%$ of the 30 non-S. aureus and S. epidermidis strains in our study were methicillin-resistant. Although $S$. epidermidis is the major coagulase-negative staphylococcal pathogen, other staphylococci such as S. lugdunensis and S. haemolyticus are important pathogens in the healthcare environment $[34,35]$.

Polymicrobial positive blood cultures generated the majority of the discordance. In contrast, the performance of BC-GP was 121/124 (98\%) in monomicrobial clinical blood cultures and 198/208 (95\%) in simulated blood cultures. In this study, polymicrobial blood cultures accounted for $14 / 106(13 \%)$ and $3 / 33$ (9\%), respectively, of the positive blood cultures at $\mathrm{TH}$ and $\mathrm{SH}$. Combined, polymicrobial cultures represented $17 / 139(12 \%)$ of the prospective clinical blood cultures which is consistent with previous studies reporting 6 to $20 \%$ of all bloodstream infections to be polymicrobial [36-38]. BC-GP correctly identified all of the organisms in $12 / 17(70 \%)$ of polymicrobial cultures. In previous BCGP studies, the correct identification rates in polymicrobial cultures ranged from 57 to $86 \%$ [14-16, 31, 32]. As misleading information can affect clinical diagnosis, resulting in inappropriate selection of antimicrobial agents, there is a need to understand the limitations of BC-GP. 


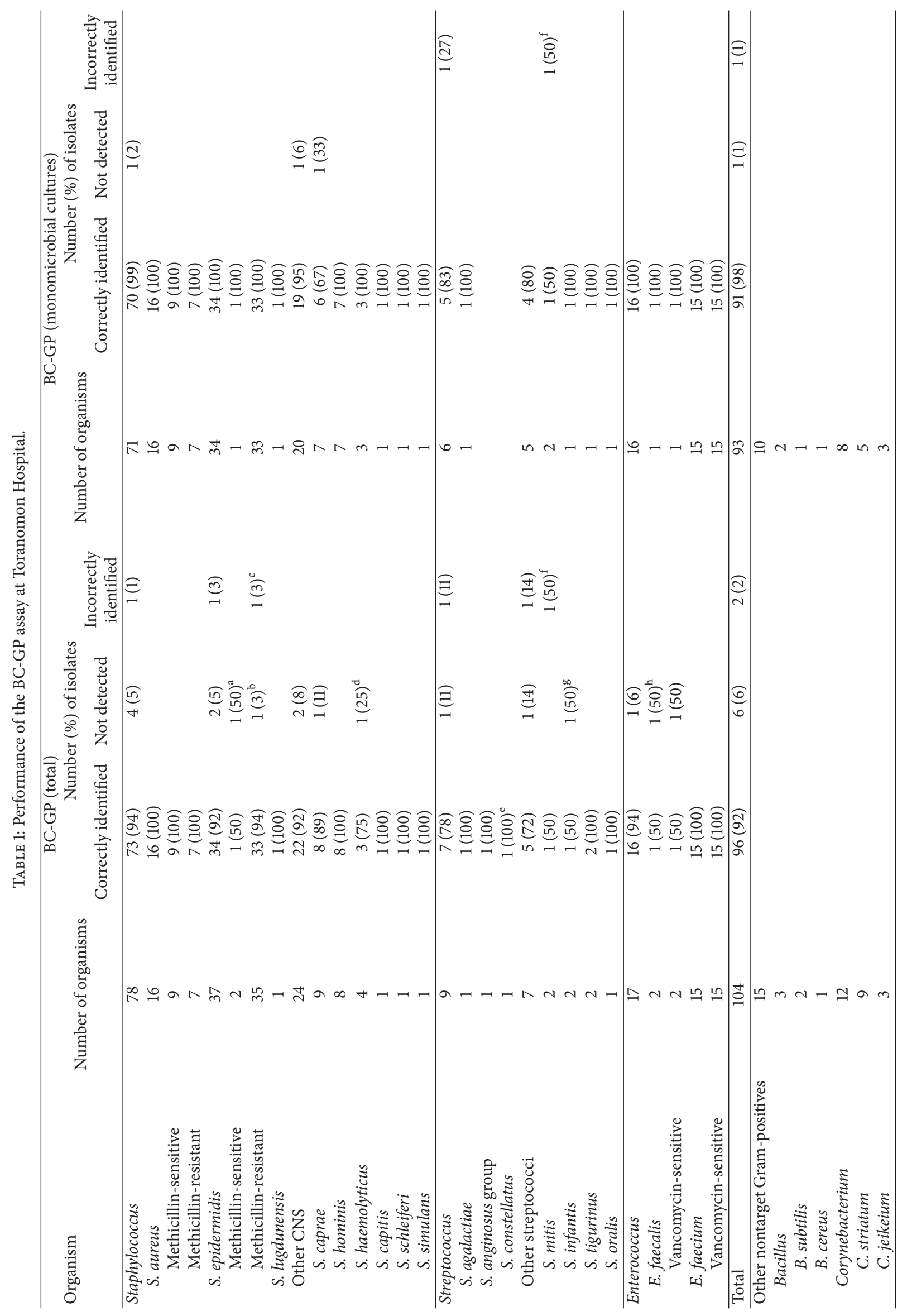




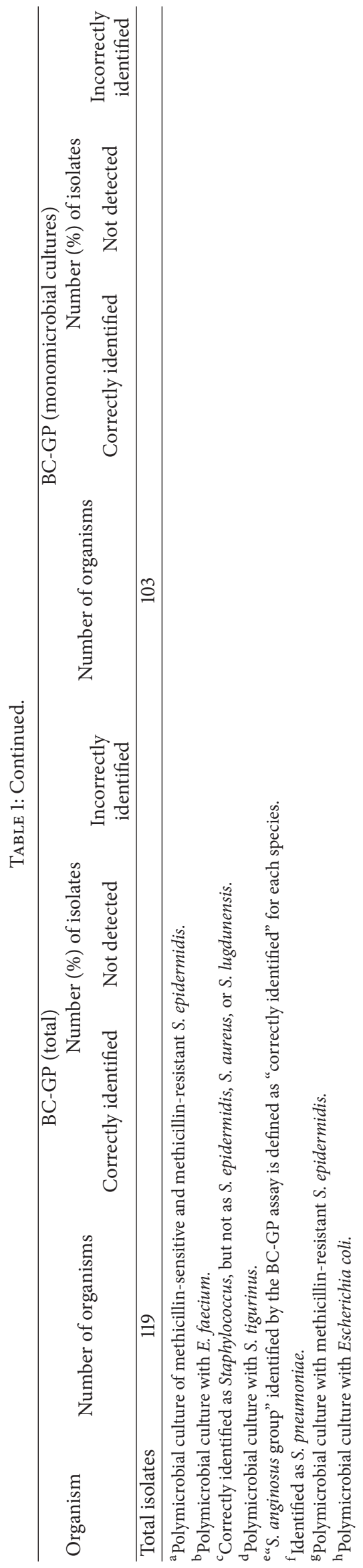




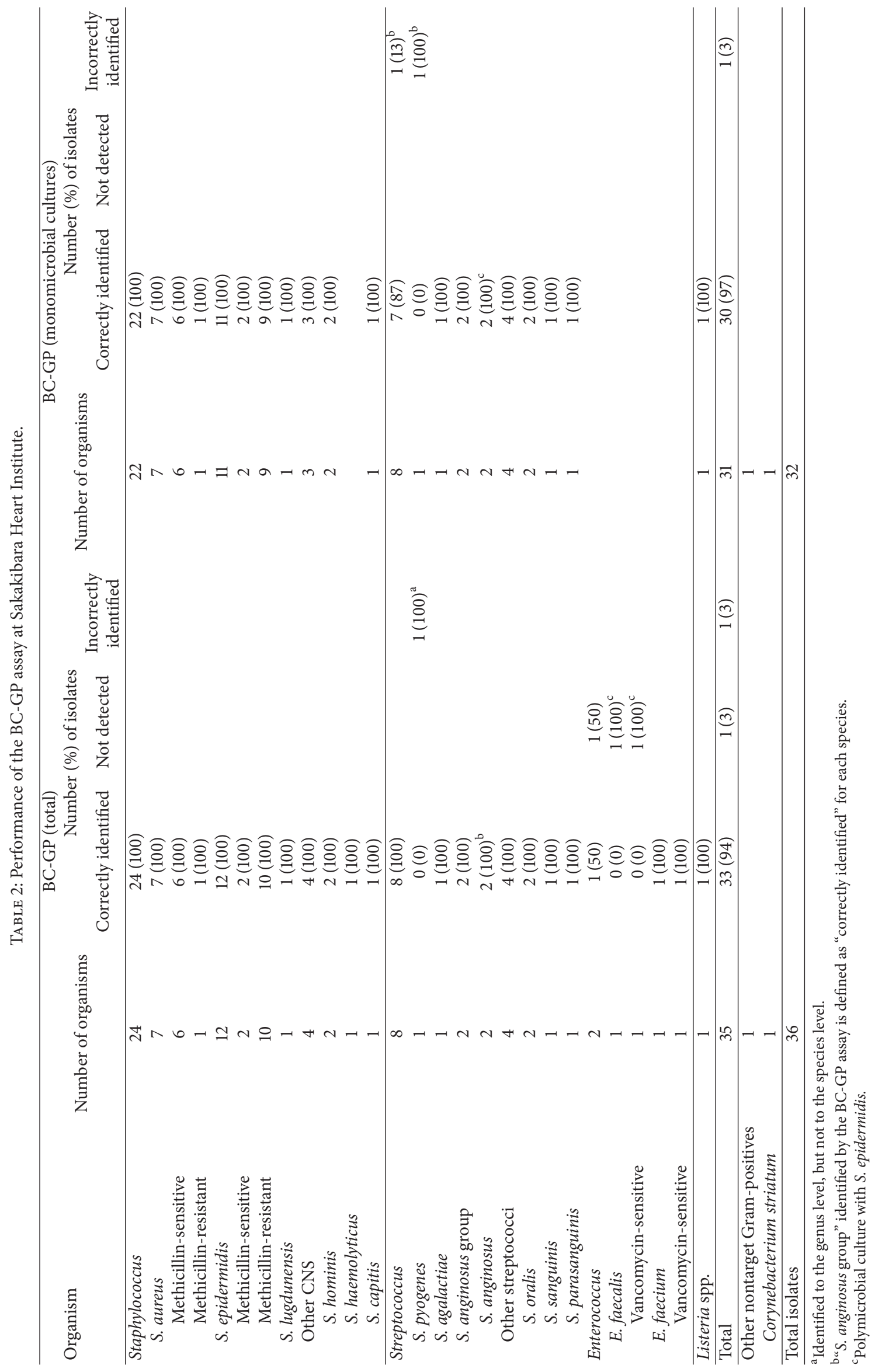


TABLE 3: Difference in time to final identification and antimicrobial susceptibility report.

\begin{tabular}{|c|c|c|c|c|}
\hline \multirow{3}{*}{ Organism } & \multicolumn{4}{|c|}{ Difference in time to result ${ }^{\mathrm{a}}$} \\
\hline & \multicolumn{2}{|c|}{ Sakakibara Heart Institute } & \multicolumn{2}{|c|}{ Toranomon Hospital } \\
\hline & Mean (h) & Range & Mean $(\mathrm{h})$ & Range \\
\hline S. aureus & $34.5(P<0.05)$ & $21.5-46.8$ & $28.2(P<0.05)$ & $19.6-47.0$ \\
\hline S. epidermidis & $80.7(P<0.05)$ & $23.9-160.9$ & $38.3(P<0.05)$ & $21.6-72.5$ \\
\hline Coagulase-negative staphylococci & $121.1(P<0.05)$ & $25.9-217.4$ & $51.4(P<0.05)$ & $21.4-72.2$ \\
\hline Enterococcus spp. & $156.6(P<0.05)$ & $95.9-217.4$ & $33.6(P<0.05)$ & $23.4-47.7$ \\
\hline Streptococcus spp. & $196.6(P<0.05)$ & $42.3-502.6$ & $51.0(P<0.05)$ & $23.4-69.2$ \\
\hline
\end{tabular}

${ }^{\mathrm{a}}$ The difference in time between BC-GP result and final culture-based identification and susceptibility results.

An additional concern with polymicrobial cultures is the clinical interpretation of $m e c A$ detection and staphylococcal detection. In our 17-polymicrobial cultures, 5 samples yielded 2 or 3 staphylococcal strains with or without mecA. This may lead to unnecessary use of vancomycin or underestimating infection caused by methicillin-resistant staphylococci. Repeating BC-GP on another set of blood cultures may lessen the risk of this problem. For monomicrobial cultures or simulated cultures, all of the discrepancies were observed with streptococci except for $1 S$. caprae strain. BCGP misidentification for streptococci included $2 S$. mitis identified as $S$. pneumoniae, no detection of $2 S$. pneumoniae, $2 S$. anginosus group, and $2 S$. pyogenes. Previous reports have also reported similar results for $S$. mitis, S. oralis, and $S$. pneumoniae [14-16, 39]. As genetic relatedness among S. mitis, S. oralis, and S. pneumoniae is well known based on $>99 \%$ homology of $16 \mathrm{~S}$ rRNA gene sequences [24], BCGP results for S. pneumoniae or Streptococcus with alphahemolysis without any positive species-specific signals should be carefully interpreted and confirmed by conventional methods such as optochin sensitivity or the bile solubility test. Interestingly, 11-fold dilution of the original blood culture medium can lead to detection of the Streptococcus signal and species signal (Table 4). A range of detection depending on the organism by BC-GP has been reported [40].

The major benefit of utilizing BC-GP is the earlier time for reporting identification and resistance determinants from positive blood cultures allowing for earlier selection of appropriate antimicrobial therapy and implementation of infection control measures such as isolation and contact precaution $[39,41]$. This has the potential of making a significant impact in the Japanese healthcare delivery system. The difference in time between BC-GP results and final culture-based identification and susceptibility results shown in Table 3 at $\mathrm{TH}$ is consistent with previous reports [14-16, 18, 32, 41]. On the other hand, earlier results of 80.7 to 196.6 hours for organisms other than S. aureus using BC-GP at SHI reflects the unavailability of clinical microbiology services during weekends and holidays. As clinical microbiology services are outsourced in many hospitals in Japan or limited to one shift during weekdays or not offered during weekends, the potential cost-benefits of retaining blood culture services in hospitals are significant. Furthermore, training of laboratory personnel in the general laboratory to recognize Grampositive cocci and bacilli will allow BC-GP testing over the weekend as well as evenings/nights. BC-GP provides the opportunity for hospitals to retain in-house a very critical laboratory service.

In conclusion, BC-GP provided accurate identification and detection of resistance markers compared with routine culture-based laboratory methods for Gram-positive organisms including CA-MRSA, HA-MRSA, and VRE strains circulating in Japan. Minimizing the time to optimizing antimicrobial therapy using BC-GP may contribute to reduced costs and improved patient care. In 2016, BC-GP received regulatory approval in Japan becoming the first multitarget molecular test for positive blood cultures approved as an in vitro diagnostic device to aid in the diagnosis of bacterial bloodstream infections. Additional studies will be necessary to validate the cost-effectiveness of BC-GP within the context of the Japanese healthcare delivery system.

\section{Ethical Approval}

This study was approved by the TH and SHI internal review boards.

\section{Competing Interests}

All the authors declare no competing interests.

\section{Authors' Contributions}

Ken Kikuchi designed and carried out the study and drafted the manuscript. Mari Matsuda, Shigekazu Iguchi, Tomonori Mizutani, Kaori Sansaka, Kenta Negishi, Kimie Shimada, Shigeyuki Notake, Hideji Yanagisawa, and Reiko Yabusaki carried out the laboratory works. Keiichi Hiramatsu, Michiru Tega-Ishii, Jun Umemura, Hiroshi Takahashi, Hideki Araoka, and Akiko Yoneyama supervised the data collection and coordinated and participated in designing the study.

\section{Acknowledgments}

This study was supported, in part, by a Grant-in-Aid (S0991013) from the Ministry of Education, Culture, Sport, 
TABLE 4: Detection of Gram-positive bacteria and resistance genes in simulated blood cultures by BC-GP.

\begin{tabular}{|c|c|c|c|c|}
\hline \multirow{2}{*}{ Organism } & \multirow{2}{*}{ Total no. of strains } & \multicolumn{3}{|c|}{ No. (\%) of isolates } \\
\hline & & correctly identified & not detected & incorrectly identified \\
\hline Staphylococcus & 54 & $54(100)$ & & \\
\hline S. aureus & 35 & $35(100)$ & & \\
\hline Methicillin-sensitive, $m e c A-$ & 15 & $15(100)$ & & \\
\hline Methicillin-resistant, $\operatorname{mec} A+$ & 20 & $20(100)$ & & \\
\hline Health-care associated & 10 & $10(100)$ & & \\
\hline Community acquired & 10 & $10(100)$ & & \\
\hline S. epidermidis & 1 & $1(100)$ & & \\
\hline Methicillin-sensitive, $m e c A-$ & 1 & $1(100)$ & & \\
\hline S. lugdunensis & 8 & $8(100)$ & & \\
\hline Other CNS & 10 & $10(100)$ & & \\
\hline S. hominis & 2 & $2(100)$ & & \\
\hline S. haemolyticus & 2 & $2(100)$ & & \\
\hline S. saprophyticus & 2 & $2(100)$ & & \\
\hline S. capitis & 1 & $1(100)$ & & \\
\hline S. cohnii & 1 & $1(100)$ & & \\
\hline S. warneri & 1 & $1(100)$ & & \\
\hline S. pseudintermedius & 1 & $1(100)$ & & \\
\hline Streptococcus & 87 & $77(88)$ & $4(5)$ & $6(7)$ \\
\hline S. pyogenes & 9 & $8(89)$ & $1(11)^{\mathrm{a}}$ & \\
\hline S. agalactiae & 8 & $8(100)$ & & \\
\hline S. dysgalactiae & 8 & $8(100)$ & & \\
\hline S. anginosus group ${ }^{\mathrm{b}}$ & 10 & $8(80)$ & & $2(20)$ \\
\hline S. anginosus & 3 & $2(66)$ & & $1(34)^{\mathrm{c}}$ \\
\hline S. constellatus & 4 & $3(75)$ & & $1(25)^{\mathrm{c}}$ \\
\hline S. intermedius & 3 & $3(100)$ & & \\
\hline S. pneumoniae & 22 & $20(91)$ & & $2(9)^{\mathrm{d}}$ \\
\hline Other streptococci & 30 & $25(86)$ & $3(10)$ & $2(7)$ \\
\hline S. mitis & 12 & $9(75)$ & $1(8)$ & $2(17)^{\mathrm{e}}$ \\
\hline S. oralis & 4 & $4(100)$ & & \\
\hline S. infantis & 2 & $2(100)$ & & \\
\hline S. mutans & 2 & $1(50)$ & $1(50)$ & \\
\hline S. sobrinus & 1 & $0(0)$ & $1(100)$ & \\
\hline S. sanguinis & 1 & $1(100)$ & & 1 \\
\hline S. parasanguinis & 1 & $1(100)$ & & \\
\hline S. peroris & 1 & $1(100)$ & & \\
\hline S. australis & 1 & $1(100)$ & & \\
\hline S. tigurinus & 1 & $1(100)$ & & \\
\hline S. cristatus & 1 & $1(100)$ & & \\
\hline S. gordonii & 1 & $1(100)$ & & \\
\hline S. gallolyticus & 1 & $1(100)$ & & \\
\hline S. lutetiensis & 1 & $1(100)$ & & \\
\hline Enterococcus & 57 & $57(100)$ & & \\
\hline E. faecalis & 32 & $32(100)$ & & \\
\hline Vancomycin-sensitive & 18 & $18(100)$ & & \\
\hline Vancomycin-resistant, vanA+ & 4 & $4(100)$ & & \\
\hline Vancomycin-resistant, vanB+ & 10 & $10(100)$ & & \\
\hline E. faecium & 25 & $25(100)$ & & \\
\hline Vancomycin-sensitive & 5 & $5(100)$ & & \\
\hline Vancomycin-resistant, vanA+ & 10 & $10(100)$ & & \\
\hline Vancomycin-resistant, vanB+ & 10 & $10(100)$ & & \\
\hline Listeria spp. & 5 & $5(100)$ & & \\
\hline
\end{tabular}


TABLE 4: Continued.

\begin{tabular}{lcccc}
\hline Organism & Total no. of strains & correctly identified & $\begin{array}{c}\text { No. (\%) of isolates } \\
\text { not detected }\end{array}$ & incorrectly identified \\
\hline Micrococcus spp. & 5 & $5(100)$ & & \\
\hline Total & 208 & $198(95)$ & $4(2)$ & $6(3)$ \\
\hline
\end{tabular}

${ }^{a}$ Not detected initially, but positive using 11-fold diluted blood culture sample.

${ }^{\mathrm{b}}$ Streptococcus sp. belonging to the S. anginosus group is identified as S. anginosus group by BC-GP.

${ }^{c}$ Positive signal for Streptococcus, but no signal for S. anginosus group.

${ }^{\mathrm{d}}$ Positive signal for Streptococcus, but no signal for S. pneumoniae.

${ }^{\mathrm{e}}$ Misidentified as S. pneumoniae.

Science, and Technology, Japan (MEXT), for the Foundation of Strategic Research Projects in Private Universities.

\section{References}

[1] P. Cappellano, C. Viscoli, P. Bruzzi, M. T. Van Lint, C. A. P. Pereira, and A. Bacigalupo, "Epidemiology and risk factors for bloodstream infections after allogeneic hematopoietic stem cell transplantion," New Microbiologica, vol. 30, no. 2, pp. 89-100, 2007.

[2] M. Mikulska, V. Del Bono, P. Bruzzi et al., "Mortality after bloodstream infections in allogeneic haematopoietic stem cell transplant (HSCT) recipients," Infection, vol. 40, no. 3, pp. 271278, 2012.

[3] M. Mikulska, V. Del Bono, R. Prinapori et al., "Risk factors for enterococcal bacteremia in allogeneic hematopoietic stem cell transplant recipients," Transplant Infectious Disease, vol. 12, no. 6, pp. 505-512, 2010.

[4] J. Vydra, R. M. Shanley, I. George et al., "Enterococcal bacteremia is associated with increased risk of mortality in recipients of allogeneic hematopoietic stem cell transplantation," Clinical Infectious Diseases, vol. 55, no. 6, pp. 764-770, 2012.

[5] T. J. Cahill and B. D. Prendergast, "Infective endocarditis," The Lancet, vol. 387, no. 10021, pp. 882-893, 2016.

[6] A. Michalopoulos, S. Geroulanos, E. S. Rosmarakis, and M. E. Falagas, "Frequency, characteristics, and predictors of microbiologically documented nosocomial infections after cardiac surgery," European Journal of Cardio-Thoracic Surgery, vol. 29, no. 4, pp. 456-460, 2006.

[7] G. S. Oderich, J. M. Panneton, T. C. Bower et al., "Infected aortic aneurysms: aggressive presentation, complicated early outcome, but durable results," Journal of Vascular Surgery, vol. 34, no. 5, pp. 900-908, 2001.

[8] C. D. Owens and K. Stoessel, "Surgical site infections: epidemiology, microbiology and prevention," Journal of Hospital Infection, vol. 70, supplement 2, pp. 3-10, 2008.

[9] B. O. Söderquist, "Surgical site infections in cardiac surgery: microbiology," APMIS, vol. 115, no. 9, pp. 1008-1011, 2007.

[10] J. Wójkowska-Mach, M. Baran, R. Drwiła et al., "Factors influencing the occurence of nosocomial bloodstream infections observed in thoracic and cardiosurgical postoperative care units," Anaesthesiology intensive therapy, vol. 44, no. 1, pp. 1620, 2012.

[11] A. Kumar, D. Roberts, B. Light et al., "Duration of hypotension before initiation of effective antimicrobial therapy is the critical determinant of survival in human septic shock," Critical Care Medicine, vol. 34, no. 6, pp. 1589-1596, 2006.

[12] B. W. Buchan, C. C. Ginocchio, R. Manii et al., "Multiplex identification of gram-positive bacteria and resistance determinants directly from positive blood culture broths: evaluation of an automated microarray-based nucleic acid test," PLoS Medicine, vol. 10, Article ID e1001478, 2013.

[13] J. Mestas, C. M. Polanco, S. Felsenstein, and J. D. Bard, "Performance of the verigene gram-positive blood culture assay for direct detection of gram-positive organisms and resistance markers in a pediatric hospital," Journal of Clinical Microbiology, vol. 52, no. 1, pp. 283-287, 2014.

[14] L. P. Samuel, R. J. Tibbetts, A. Agotesku, M. Fey, R. Hensley, and F. A. Meier, "Evaluation of a microarray-based assay for rapid identification of gram-positive organisms and resistance markers in positive blood cultures," Journal of Clinical Microbiology, vol. 51, no. 4, pp. 1188-1192, 2013.

[15] K. V. Sullivan, N. N. Turner, S. S. Roundtree et al., "Rapid detection of gram-positive organisms by use of the verigene grampositive blood culture nucleic acid test and the bact/alert pediatric fan system in a multicenter pediatric evaluation," Journal of Clinical Microbiology, vol. 51, no. 11, pp. 3579-3584, 2013.

[16] C. M. Wojewoda, L. Sercia, M. Navas et al., "Evaluation of the verigene gram-positive blood culture nucleic acid test for rapid detection of bacteria and resistance determinants," Journal of Clinical Microbiology, vol. 51, no. 7, pp. 2072-2076, 2013.

[17] H. Suzuki, S. Hitomi, Y. Yaguchi et al., "Prospective intervention study with a microarray-based, multiplexed, automated molecular diagnosis instrument (Verigene system) for the rapid diagnosis of bloodstream infections, and its impact on the clinical outcomes," Journal of Infection and Chemotherapy, vol. 21, no. 12, pp. 849-856, 2015.

[18] M. Dodémont, R. De Mendonça, C. Nonhoff, S. Roisin, and O. Denis, "Evaluation of Verigene Gram-positive blood culture assay performance for bacteremic patients," European Journal of Clinical Microbiology and Infectious Diseases, vol. 34, no. 3, pp. 473-477, 2014.

[19] G. K. H. Siu, J. H. K. Chen, T. K. Ng et al., "Performance evaluation of the verigene gram-positive and gram-negative blood culture test for direct identification of bacteria and their resistance determinants from positive blood cultures in Hong Kong," PLOS ONE, vol. 10, no. 10, Article ID e0139728, 2015.

[20] Japan Nosocomial Infections Surveillance (JANIS), "Open Report 2013-Hospital-acquired Infection Surveillance," 2014, https://www.nih-janis.jp/english/report/open_report/2013/3/ 1ken_Open_Report_Eng_201300.pdf.

[21] Clinical and Laboratory Standards Institute, Performance Standards for Antimicrobial Susceptibility Testing, Twenty-First Informational Supplement M100-S21, Clinical and Laboratory Standards Institute, Wayne, Pa, USA, 2011. 
[22] Y. Nakatomi and J. Sugiyama, "A rapid latex agglutination assay for the detection of penicillin-binding protein $2^{\prime}$," Microbiology and Immunology, vol. 42, no. 11, pp. 739-743, 1998.

[23] N. Matsuda, M. Matsuda, S. Notake et al., "Evaluation of a simple protein extraction method for species identification of clinically relevant staphylococci by matrix-assisted laser desorption ionization-time of flight mass spectrometry," Journal of Clinical Microbiology, vol. 50, no. 12, pp. 3862-3866, 2012.

[24] Y. Kawamura, X.-G. Hou, F. Sultana, H. Miura, and T. Ezaki, "Determination of 16S rRNA sequences of Streptococcus mitis and Streptococcus gordonii and phylogenetic relationships among members of the genus Streptococcus," International Journal of Systematic Bacteriology, vol. 45, no. 2, pp. 406-408, 1995.

[25] Y. Kawamura, R. A. Whiley, S.-E. Shu, T. Ezaki, and J. M. Hardie, "Genetic approaches to the identification of the mitis group within the genus Streptococcus," Microbiology, vol. 145, no. 9, pp. 2605-2613, 1999.

[26] C. Poyart, G. Quesne, C. Boumaila, and P. Trieu-Cuot, "Rapid and accurate species-level identification of coagulase-negative staphylococci by using the sodA gene as a target," Journal of Clinical Microbiology, vol. 39, no. 12, pp. 4296-4301, 2001.

[27] K. Murakami, W. Minamide, K. Wada, E. Nakamura, H. Teraoka, and S. Watanabe, "Identification of methicillin-resistant strains of staphylococci by polymerase chain reaction," Journal of Clinical Microbiology, vol. 29, no. 10, pp. 2240-2244, 1991.

[28] S. Dutka-Malen, S. Evers, and P. Courvalin, "Detection of glycopeptide resistance genotypes and identification to the species level of clinically relevant enterococci by PCR," Journal of Clinical Microbiology, vol. 33, no. 1, pp. 24-27, 1995.

[29] Y. Kondo, T. Ito, X. X. Ma et al., "Combination of multiplex PCRs for staphylococcal cassette chromosome mec type assignment: rapid identification system for $m e c, c c r$, and major differences in junkyard regions," Antimicrobial Agents and Chemotherapy, vol. 51, no. 1, pp. 264-274, 2007.

[30] C. Piao, T. Karasawa, K. Totsuka, T. Uchiyama, and K. Kikuchi, "Prospective surveillance of community-onset and healthcareassociated methicillin-resistant Staphylococcus aureus isolated from a university-affiliated hospital in Japan," Microbiology and Immunology, vol. 49, no. 11, pp. 959-970, 2005.

[31] S. L. Aitken, V. S. Hemmige, H. L. Koo, N. N. Vuong, T. M. Lasco, and K. W. Garey, "Real-world performance of a microarray-based rapid diagnostic for Gram-positive bloodstream infections and potential utility for antimicrobial stewardship," Diagnostic Microbiology and Infectious Disease, vol. 81, no. 1, pp. 4-8, 2015.

[32] S. G. Beal, J. Ciurca, G. Smith et al., "Evaluation of the nanosphere verigene gram-positive blood culture assay with the versaTREK blood culture system and assessment of possible impact on selected patients," Journal of Clinical Microbiology, vol. 51, no. 12, pp. 3988-3992, 2013.

[33] R. M. Martinez, E. R. Bauerle, F. C. Fang, and S. M. Butler$\mathrm{Wu}$, "Evaluation of three rapid diagnostic methods for direct identification of microorganisms in positive blood cultures," Journal of Clinical Microbiology, vol. 52, no. 7, pp. 2521-2529, 2014.

[34] K. L. Frank, J. L. Del Pozo, and R. Patel, "From clinical microbiology to infection pathogenesis: how daring to be different works for staphylococcus lugdunensis," Clinical Microbiology Reviews, vol. 21, no. 1, pp. 111-133, 2008.

[35] E. M. Barros, H. Ceotto, M. C. F. Bastos, K. R. N. Dos Santos, and M. Giambiagi-deMarval, "Staphylococcus haemolyticus as an important hospital pathogen and carrier of methicillin resistance genes," Journal of Clinical Microbiology, vol. 50, no. 1, pp. 166-168, 2012.

[36] G. S. Cooper, D. S. Havlir, D. M. Shlaes, and R. A. Salata, "Polymicrobial bacteremia in the late 1980s: predictors of outcome and review of the literature," Medicine, vol. 69, no. 2, pp. 114-123, 1990.

[37] S. Sancho, A. Artero, R. Zaragoza, J. J. Camarena, R. González, and J. M. Nogueira, "Impact of nosocomial polymicrobial bloodstream infections on the outcome in critically ill patients," European Journal of Clinical Microbiology and Infectious Diseases, vol. 31, no. 8, pp. 1791-1796, 2012.

[38] S. Y. Park, K.-H. Park, K. M. Bang et al., "Clinical significance and outcome of polymicrobial Staphylococcus aureus bacteremia," The Journal of Infection, vol. 65, no. 2, pp. 119-127, 2012.

[39] K. Alby, L. M. Daniels, D. J. Weber, and M. B. Miller, "Development of a treatment algorithm for streptococci and enterococci from positive blood cultures identified with the Verigene grampositive blood culture assay," Journal of Clinical Microbiology, vol. 51, no. 11, pp. 3869-3871, 2013.

[40] T. Raich and S. Powell, "Identification of bacterial and fungal pathogens from positive blood culture bottles: a microarraybased approach," Methods in Molecular Biology, vol. 1237, pp. 73-90, 2015.

[41] D. G. Roshdy, A. Tran, N. LeCroy et al., "Impact of a rapid microarray-based assay for identification of positive blood cultures for treatment optimization for patients with streptococcal and enterococcal bacteremia," Journal of Clinical Microbiology, vol. 53, no. 4, pp. 1411-1414, 2015. 


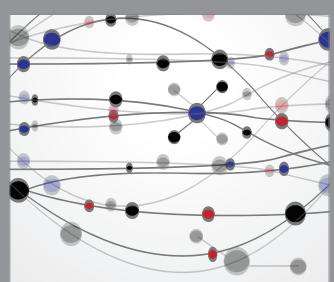

The Scientific World Journal
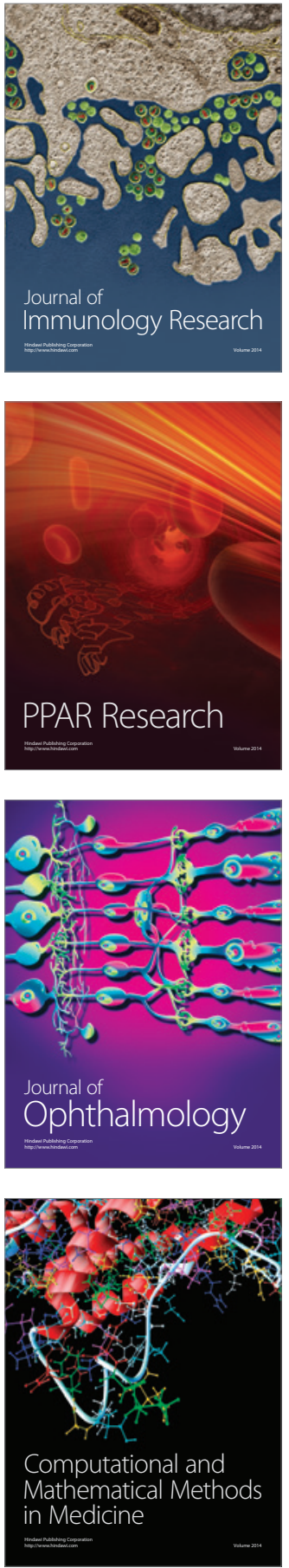

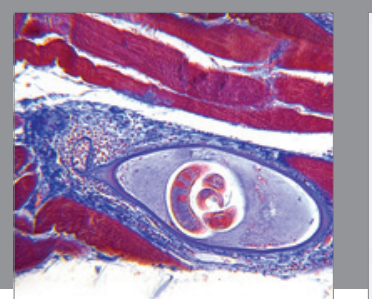

Gastroenterology Research and Practice
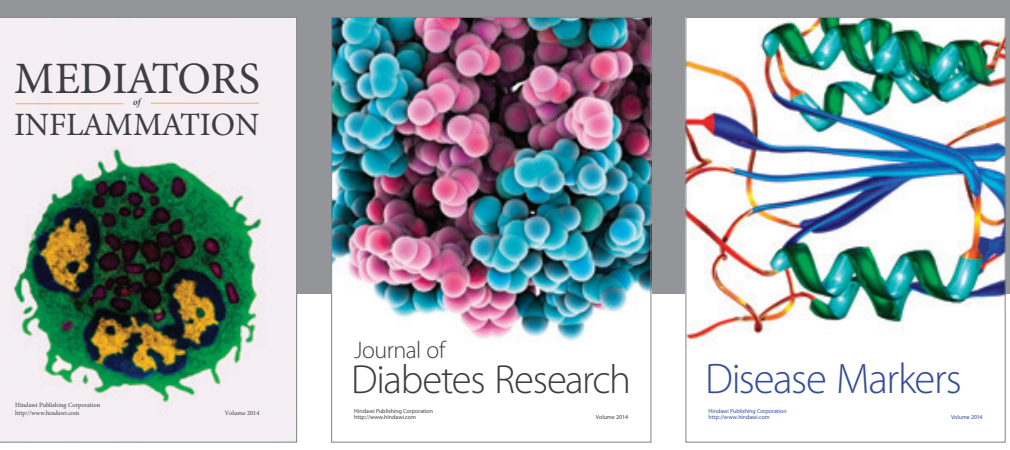

Disease Markers

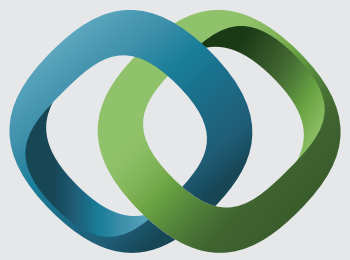

\section{Hindawi}

Submit your manuscripts at

https://www.hindawi.com
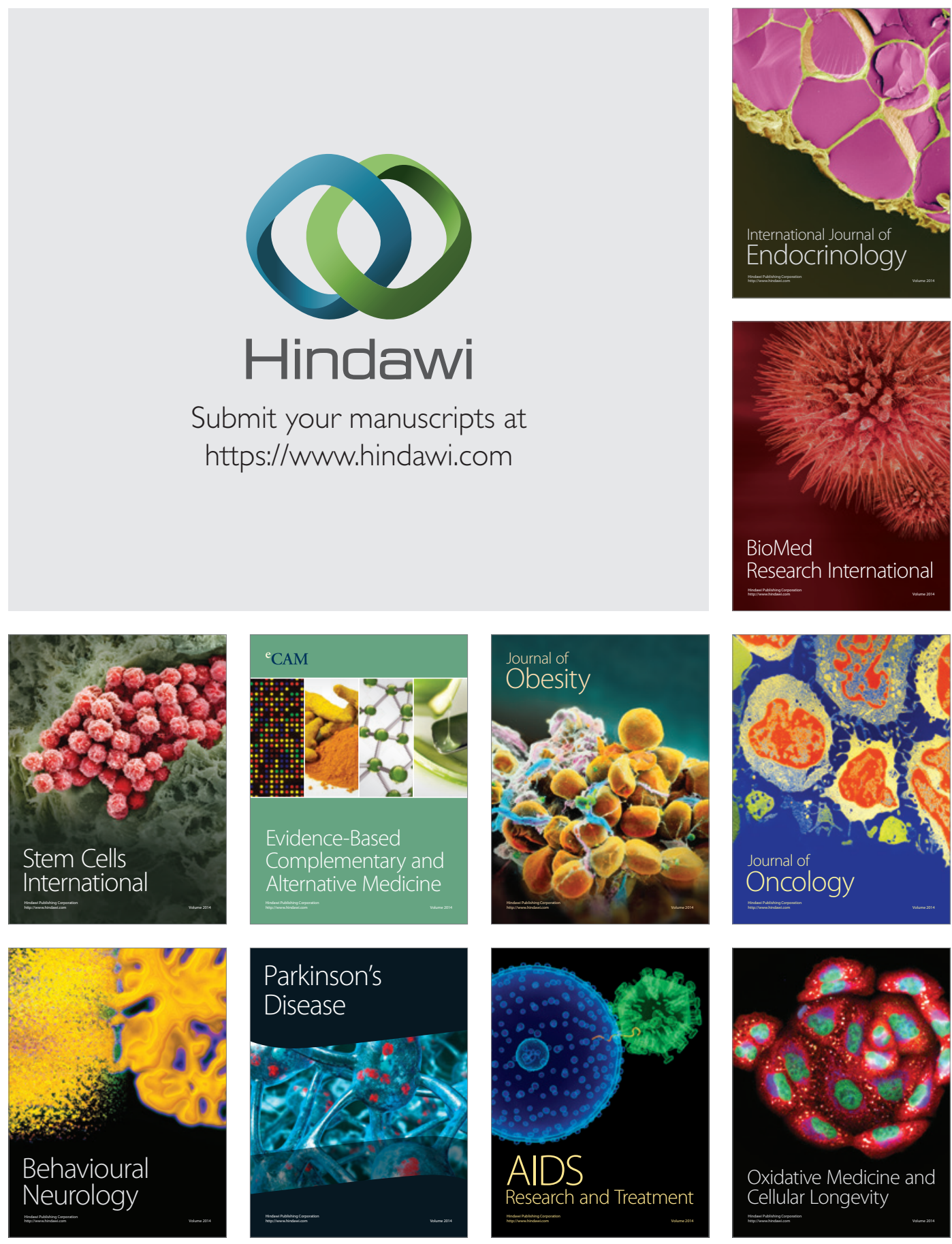